gap> $\mathrm{g}:=$ SymmetricGroup ( 4 );

$\operatorname{Sym}([1 \ldots 4])$

i5 : betti $(t$, Weights $\Rightarrow\{1$, gap $\}$

false

01234 gap> tblmod2:= CharacterTable( tbl, 2);

05 = total: 1413144 BrauerTable $(\operatorname{Sym}([1 \ldots 4]), 2)$

1: . 2242 gap> tblmod2 = CharacterTable $(t b l, 2)$;

Journal of Software for

01234 gap> libtbl:= CharacterTable( "M" );

Algebra and Geometry

fail $\quad r i n g ~ r 1=32003,(x, y, z)$, ds;

o6 : BettiTally 1 CharacterTable ( "Sym (4)" )

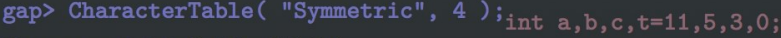

gap> ComputedBrauerTables ( tbl );

$\begin{array}{lllll}0 & 1 & 2 & 3 & 4\end{array}$

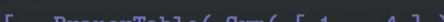
poly $f=\mathrm{x}^{\wedge} \mathrm{a}+\mathrm{y}^{\wedge} \mathrm{b}+\mathrm{z}^{\wedge}(3 * \mathrm{c})+\mathrm{x}^{\wedge}(\mathrm{c}+2) * \mathrm{y}^{\wedge}(\mathrm{c}-1)+\mathrm{x}^{\wedge}$ $\mathrm{x}^{\sim}(\mathrm{c}-2) * \mathrm{y}^{\sim} \mathrm{c} *\left(\mathrm{y}^{\sim} 2+\mathrm{t} * \mathrm{x}\right)^{\sim}-2$;

o7 = total: 1413144

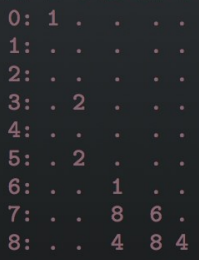

o7 : BettiTally

i8 : peek t1

$08=$ BettiTally $\{(0,\{0,0\}, 0) \Rightarrow 1\}$

$(1,\{2,2\}, 4) \Rightarrow 2$

(1, $\{3,3\}, 6) \Rightarrow 2$

(2, $\{3,7\}, 10) \Rightarrow 2$

$(2,\{4,4\}, 8) \Rightarrow 1$

(2, $\{4,5\}, 9) \Rightarrow 4$

$(2,\{5,4\}, 9) \Rightarrow 4$

(2, $\{7,3\}, 10) \Rightarrow 2$

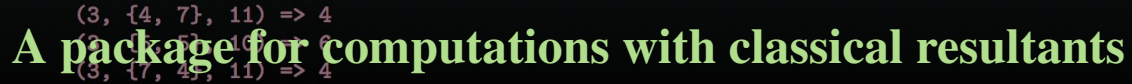

$(4,\{5,7\}, 12) \Rightarrow 2$

$(4,\{7,5\}, 12) \Rightarrow 2$ GIOVANNI STAGLIANÒ 


\title{
A package for computations with classical resultants
}

\author{
GiOVANNI STAGLIANÒ
}

\begin{abstract}
We present the Macaulay2 package Resultants, which provides commands for the effective computation of multivariate resultants, discriminants, and Chow forms. We provide some background for the algorithms implemented and show, with a few examples, how the package works.
\end{abstract}

INTRODUCTION. The resultant characterizes the existence of nontrivial solutions for a square system of homogeneous polynomial equations as a condition on the coefficients. One of its important features is that it can be used to compute elimination ideals and to solve polynomial equations. Indeed, it provides one of the two main tools in elimination theory, along with Gröbner bases. The resultant of the system of equations given by the partial derivatives of a complex homogeneous polynomial $F$ is called (up to a constant factor) the discriminant of $F$. It characterizes the existence of singular points in the projective hypersurface $V(F)$ as a condition on the coefficients of $F$. In this special case, all polynomial equations have the same total degree. Every time the system of equations consists of $n+1$ polynomial equations of the same total degree $d$, the resultant has a further interesting property: it can be expressed as a polynomial of degree $d^{n}$ in the $(n+1) \times(n+1)$ minors of an $(n+1) \times\left(\begin{array}{c}n+d \\ n\end{array}\right)$ matrix, the coefficient matrix of the system of equations. This allows us to write down a generic resultant in a more compact form. The polynomial of degree $d^{n}$ so obtained is geometrically interpreted as the Chow form of the $d$-th Veronese embedding of $\mathbb{P}^{n}$.

The package Resultants, included with [Macaulay2], provides commands for the explicit computation of resultants and discriminants. The main algorithm used is based on the so-called Poisson formula, which reduces the computation of the resultant of $n+1$ equations to the product of the resultant of $n$ equations with the determinant of an appropriate matrix. This algorithm requires a certain genericity condition on the input polynomials, achievable with a generic change of coordinates. The package also includes tools for working with Chow forms and more generally with tangential Chow forms.

MSC2010: primary 13P15; secondary 68W30.

Keywords: resultant, discriminant, Chow form.

Resultants version 1.2.1 
In Section 1, from a more computational point of view, we give some background information on the general theory of resultants, discriminants, and Chow forms. In Section 2, we briefly illustrate how to use the package with the help of some examples; more detailed information and examples can be found in its documentation.

1. OVERVIEW OF CLASSICAL RESUlTANTS. We present an overview of some classically well known facts on the theory of resultants for forms in several variables. For details and proofs, we refer mainly to [Gelfand et al. 1994; Cox et al. 2005]; other references are [Jouanolou 1991; 1997; van der Waerden 1950; Demazure 2012; Emiris and Mourrain 1999; Bajaj et al. 1988; Busé and Jouanolou 2014], and [Cox et al. 2007] for the case of two bivariate polynomials.

Resultants. Suppose we are given $n+1$ homogeneous polynomials $F_{0}, \ldots, F_{n}$ in $n+1$ variables $x_{0}, \ldots, x_{n}$ over the complex field $\mathbb{C}$. For $i=0, \ldots, n$, let $d_{i}$ denote the total degree of $F_{i}$ so that we can write $F_{i}=\sum_{|\alpha|=d_{i}} c_{i, \alpha} x^{\alpha}$, where $x^{\alpha}$ denotes $x_{0}^{\alpha_{0}} \cdots x_{n}^{\alpha_{n}}$. For each pair of indices $i, \alpha$, we introduce a variable $u_{i, \alpha}$ and form the universal ring of coefficients $\mathbb{U}_{d_{0}, \ldots, d_{n}}:=\mathbb{Z}\left[u_{i, \alpha}: i=0, \ldots, n,|\alpha|=d_{i}\right]$. If $P \in \mathbb{U}_{d_{0}, \ldots, d_{n}}$, we denote by $P\left(F_{0}, \ldots, F_{n}\right)$ the element in $\mathbb{C}$ obtained by replacing each variable $u_{i, \alpha}$ with the corresponding coefficient $c_{i, \alpha}$.

Theorem 1.1 [Gelfand et al. 1994; Cox et al. 2005]. If we fix positive degrees $d_{0}, \ldots, d_{n}$, then there is a unique polynomial $\operatorname{Res}=\operatorname{Res}_{d_{0}, \ldots, d_{n}} \in \mathbb{U}_{d_{0}, \ldots, d_{n}}$ which has the following properties:

(1) If $F_{0}, \ldots, F_{n} \in \mathbb{C}\left[x_{0}, \ldots, x_{n}\right]$ are homogeneous of degrees $d_{0}, \ldots, d_{n}$, then the equations

$$
F_{0}=0, \ldots, F_{n}=0
$$

have a nontrivial solution over $\mathbb{C}\left(\right.$ i.e., $\left.\varnothing \neq V\left(F_{0}, \ldots, F_{n}\right) \subset \mathbb{P}_{\mathbb{C}}^{n}\right)$ if and only if $\operatorname{Res}\left(F_{0}, \ldots, F_{n}\right)=0$.

(2) Res is irreducible, even when regarded as a polynomial over $\mathbb{C}$.

(3) $\operatorname{Res}\left(x_{0}^{d_{0}}, \ldots, x_{n}^{d_{n}}\right)=1$.

Definition 1.2. We call $\operatorname{Res}\left(F_{0}, \ldots, F_{n}\right)$ the resultant of $F_{0}, \ldots, F_{n}$.

Remark 1.3. If $A$ is any commutative ring, we define the resultant of $n+1$ homogeneous polynomials $F_{0}, \ldots, F_{n} \in A\left[x_{0}, \ldots, x_{n}\right]$ again as $\operatorname{Res}\left(F_{0}, \ldots, F_{n}\right) \in A$, i.e., by specializing the coefficients of the integer polynomial Res. Thus, the formation of resultants commutes with specialization.

Example 1.4. The resultant is a direct generalization of the determinant. Indeed, if $d_{0}=\cdots=d_{n}=1$, then $\operatorname{Res}\left(F_{0}, \ldots, F_{n}\right)$ equals the determinant of the $(n+1) \times$ $(n+1)$ coefficient matrix. 
Proposition 1.5 [Jouanolou 1991; Jouanolou 1997]. The following hold:

(1) (homogeneity) For a fixed $j$ between 0 and $n$, Res is homogeneous in the variables $u_{j, \alpha},|\alpha|=d_{j}$, of degree $d_{0} \cdots d_{j-1} d_{j+1} \cdots d_{n}$; hence its total degree is $\sum_{j=0}^{n} d_{0} \cdots d_{j-1} d_{j+1} \cdots d_{n}$.

(2) (symmetry) If $\sigma$ is a permutation of $\{0, \ldots, n\}$, then

$$
\operatorname{Res}\left(F_{\sigma(0)}, \ldots, F_{\sigma(n)}\right)=\operatorname{sign}(\sigma)^{d_{0} \cdots d_{n}} \operatorname{Res}\left(F_{0}, \ldots, F_{n}\right) .
$$

(3) (multiplicativity) If $F_{j}=F_{j}^{\prime} F_{j}^{\prime \prime}$, then we have

$\operatorname{Res}\left(F_{0}, \ldots, F_{j}, \ldots, F_{n}\right)=\operatorname{Res}\left(F_{0}, \ldots, F_{j}^{\prime}, \ldots, F_{n}\right) \operatorname{Res}\left(F_{0}, \ldots, F_{j}^{\prime \prime}, \ldots, F_{n}\right)$.

(4) $(\operatorname{SL}(n+1)$-invariance) For each $(n+1) \times(n+1)$ matrix $A$ over $\mathbb{C}$, we have

$$
\operatorname{Res}\left(F_{0}(A x), \ldots, F_{n}(A x)\right)=\operatorname{det}(A)^{d_{0} \cdots d_{n}} \operatorname{Res}\left(F_{0}(x), \ldots, F_{n}(x)\right),
$$

where Ax denotes the product of $A$ with the column vector $\left(x_{0}, \ldots, x_{n}\right)^{t}$.

(5) (elementary transformation) If $H_{i}$ is homogeneous of degree $d_{j}-d_{i}$, then

$$
\operatorname{Res}\left(F_{0}, \ldots, F_{j}+\sum_{i \neq j} H_{i} F_{i}, \ldots, F_{n}\right)=\operatorname{Res}\left(F_{0}, \ldots, F_{j}, \ldots, F_{n}\right) .
$$

Remark 1.6. On the product $\mathbb{A}^{M} \times \mathbb{P}^{n}=\operatorname{Spec}\left(\mathbb{C}\left[u_{i, \alpha}\right]\right) \times \operatorname{Proj}\left(\mathbb{C}\left[x_{0}, \ldots, x_{n}\right]\right)$, where $M=\sum_{i=0}^{n}\left(\begin{array}{c}n+d_{i} \\ n\end{array}\right)$, we have an incidence variety

$$
W:=\left\{\left(\left(c_{i, \alpha}\right), p\right) \in \mathbb{A}^{M} \times \mathbb{P}^{n}: p \in V\left(\sum_{|\alpha|=d_{0}} c_{0, \alpha} x^{\alpha}, \ldots, \sum_{|\alpha|=d_{n}} c_{n, \alpha} x^{\alpha}\right)\right\} .
$$

The first projection $\pi_{1}: W \rightarrow \mathbb{A}^{M}$ is birational onto its image, whereas all the fibers of the second projection $\pi_{2}: W \rightarrow \mathbb{P}^{n}$ are linear subspaces of dimension $M-n-1$. It follows that $W$ is a smooth irreducible variety which is birational to $\overline{\pi_{1}(W)}=\pi_{1}(W)=V\left(\operatorname{Res}_{d_{0}, \ldots, d_{n}}\right) \subset \mathbb{A}^{M}$.

The following result is called the Poisson formula and allows one to compute resultants inductively.

Theorem 1.7 [Jouanolou 1991; Cox et al. 2005]. Let

$$
f_{i}\left(x_{0}, \ldots, x_{n-1}\right):=F_{i}\left(x_{0}, \ldots, x_{n-1}, 1\right)
$$

and $\overline{F_{i}}\left(x_{0}, \ldots, x_{n-1}\right):=F_{i}\left(x_{0}, \ldots, x_{n-1}, 0\right)$. If $\operatorname{Res}\left(\overline{F_{0}}, \ldots, \overline{F_{n-1}}\right) \neq 0$, then the quotient ring $A=\mathbb{C}\left[x_{0}, \ldots, x_{n-1}\right] /\left(f_{0}, \ldots, f_{n-1}\right)$ has dimension $d_{0} \cdots d_{n-1}$ as a vector space over $\mathbb{C}$, and

$$
\operatorname{Res}\left(F_{0}, \ldots, F_{n}\right)=\operatorname{Res}\left(\overline{F_{0}}, \ldots, \overline{F_{n-1}}\right)^{d_{n}} \operatorname{det}\left(m_{f_{n}}: A \rightarrow A\right),
$$

where $m_{f_{n}}: A \rightarrow A$ is the linear map given by multiplication by $f_{n}$. 
With the same hypotheses as Theorem 1.7, a monomial basis for $A$ over $\mathbb{C}$ (useful in the implementation) can be constructed as explained in [Cox et al. 2005, Chapter 2, §2]. Note also that we have

$$
\operatorname{det}\left(m_{f_{n}}: A \rightarrow A\right)=\prod_{p \in V} f_{n}(p)^{\operatorname{mult}_{p}(V)},
$$

where $V=V\left(f_{0}, \ldots, f_{n-1}\right)$.

We now describe the most popular way to compute resultants, which is due to Macaulay [1903]. Let

$$
\delta=\sum_{i=0}^{n} d_{i}-n \quad \text { and } \quad N=\left(\begin{array}{c}
n+\delta \\
n
\end{array}\right) .
$$

We can divide the monomials $x^{\alpha}$ of total degree $\delta$ into the $n+1$ mutually disjoint sets

$$
S_{i}:=\left\{x^{\alpha}:|\alpha|=\delta, \min \left\{j: x_{j}^{d_{j}} \mid x^{\alpha}\right\}=i\right\}, \quad \text { for } i=0, \ldots, n .
$$

A monomial $x^{\alpha}$ of total degree $\delta$ is called reduced if $x_{i}^{d_{i}}$ divides $x^{\alpha}$ for exactly one $i$. Consider the following $N$ homogeneous polynomials of degree $\delta$ :

$$
x^{\alpha} / x_{i}^{d_{i}} F_{i}, \quad \text { for } i=0, \ldots n \text { and } x^{\alpha} \in S_{i} .
$$

By regarding the monomials of total degree $\delta$ as unknowns, the polynomials in (1-3) form a system of $N$ linear equations in $N$ unknowns. Let

$$
\mathbb{D}=\mathbb{D}\left(F_{0}, \ldots, F_{n}\right)
$$

denote the coefficient matrix of this linear system, and let $\mathbb{D}^{\prime}\left(F_{0}, \ldots, F_{n}\right)$ denote the submatrix of $\mathbb{D}$ obtained by deleting all rows and columns corresponding to reduced monomials. The following result is called the Macaulay formula and allows one to compute the resultant as a quotient of two determinants.

Theorem 1.8 [MacAulay 1903; Jouanolou 1997; Cox et al. 2005]. The following formula holds:

$$
\operatorname{det}\left(\mathbb{D}\left(F_{0}, \ldots, F_{n}\right)\right)=\operatorname{Res}\left(F_{0}, \ldots, F_{n}\right) \operatorname{det}\left(\mathbb{D}^{\prime}\left(F_{0}, \ldots, F_{n}\right)\right) .
$$

In several special cases, the resultant can be expressed as a single determinant (see [Gelfand et al. 1994, Chapter 13, Proposition 1.6]). We also mention that besides (1-4), there are other ways to represent resultants as quotients: these include Bezoutians [Elkadi and Mourrain 1998] and Dixon matrices [Kapur et al. 1994]; see also [Emiris and Mourrain 1999] and [Cox et al. 2005, p. 110]. However, all these matrices are usually of much larger size than those involved by the Poisson formula (1-1), as shown in the following simple example (see [Emiris and Mourrain 1999], for a comparison between Macaulay and other resultant matrices). 
Example 1.9. Let $F_{0}=x^{3}+y^{2} z, F_{1}=x y+y^{2}+x z+y z, F_{2}=y^{4}+z^{4} \in \mathbb{C}[x, y, z]$. The Poisson formula expresses $\operatorname{Res}\left(F_{0}, F_{1}, F_{2}\right)$ as the following product of determinants:

$$
\operatorname{Res}\left(F_{0}, F_{1}, F_{2}\right)=\left(1^{2} \operatorname{det}\left(\begin{array}{lll}
1 & 0 & 0 \\
1 & 1 & 0 \\
0 & 1 & 1
\end{array}\right)\right)^{4} \cdot \operatorname{det}\left(\begin{array}{cccccc}
1 & 0 & 0 & 0 & 0 & 0 \\
0 & 2 & 0 & 0 & 0 & 0 \\
0 & 0 & 2 & 0 & 0 & 0 \\
0 & 1 & 0 & 1 & 0 & 0 \\
1 & 0 & 0 & 0 & 2 & 0 \\
0 & -1 & 0 & 1 & 0 & 2
\end{array}\right)=16
$$

The Macaulay formula yields the same result as a quotient $\operatorname{det}(\mathbb{D}) / \operatorname{det}\left(\mathbb{D}^{\prime}\right)$, where $\mathbb{D}$ and $\mathbb{D}^{\prime}$ are square matrices of size $36 \times 36$ and $10 \times 10$, respectively.

Discriminants. Let $F=\sum_{|\alpha|=d} c_{\alpha} x^{\alpha} \in \mathbb{C}\left[x_{0}, \ldots, x_{n}\right]$ be a homogeneous polynomial of a certain degree $d$. As above, for each index $\alpha$ we introduce a variable $u_{\alpha}$ and form the universal ring of coefficients $\mathbb{U}_{d}:=\mathbb{C}\left[u_{\alpha}:|\alpha|=d\right]$. Then one can show that, up to sign, there is a unique polynomial Disc $=\operatorname{Disc}_{d} \in \mathbb{U}_{d}$ which has the following properties:

(1) If $F \in \mathbb{C}\left[x_{0}, \ldots, x_{n}\right]$ is homogeneous of degrees $d$, then the equations

$$
\partial F / \partial x_{0}=0, \ldots, \partial F / \partial x_{n}=0
$$

have a nontrivial solution over $\mathbb{C}$ (i.e., the hypersurface defined by $F$ is singular) if and only if $\operatorname{Disc}(F)=0$;

(2) Disc is irreducible, even when regarded as a polynomial over $\mathbb{C}$.

Proposition 1.10 [Gelfand et al. 1994]. Up to sign, we have the formula

$$
\operatorname{Disc}(F)=c_{d, n} \operatorname{Res}\left(\frac{\partial F}{\partial x_{0}}, \ldots, \frac{\partial F}{\partial x_{n}}\right) \text {, where } c_{d, n}=d^{\frac{(-1)^{n+1}-(d-1)^{n+1}}{d}} .
$$

Definition 1.11. We call the polynomial defined by (1-5) the discriminant of $F$.

Proposition 1.12 [Gelfand et al. 1994]. The following hold:

(1) The polynomial Disc is homogeneous of degree $(n+1)(d-1)^{n}$.

(2) For each $(n+1) \times(n+1)$ matrix A over $\mathbb{C}$, we have

$$
\operatorname{Disc}(F(A x))=\operatorname{det}(A)^{d(d-1)^{n}} \operatorname{Disc}(F(x)),
$$

where Ax denotes the product of $A$ with the column vector $\left(x_{0}, \ldots, x_{n}\right)^{t}$.

Geometrically, we have the following interpretation.

Proposition 1.13 [Gelfand et al. 1994]. The discriminant hypersurface $V\left(\right.$ Disc $\left._{d}\right)$ in the space of forms of degree $d$ on $\mathbb{P}^{n}$ coincides with the dual variety of the $d$-th Veronese embedding of $\mathbb{P}^{n}$. 
Chow forms. Let $X \subset \mathbb{P}^{n}$ be an irreducible subvariety of dimension $k$ and degree $d$. Consider the subvariety $Z(X)$ in the Grassmannian $\mathbb{G}\left(n-k-1, \mathbb{P}^{n}\right)$ of all $(n-k-1)$ dimensional projective subspaces of $\mathbb{P}^{n}$ that intersect $X$. It turns out that $Z(X)$ is an irreducible hypersurface of degree $d$; thus $Z(X)$ is defined by the vanishing of some element $R_{X}$, unique up to a constant factor, in the homogeneous component of degree $d$ of the coordinate ring of the Grassmannian $\mathbb{G}\left(n-k-1, \mathbb{P}^{n}\right)$ in the Plücker embedding. This element is called the Chow form of $X$. It is notable that $X$ can be recovered from its Chow form. See [Gelfand et al. 1994, Chapter 3, §2] for details.

Consider the product $\mathbb{P}^{k} \times X$ as a subvariety of $\mathbb{P}^{(k+1)(n+1)-1}$ via the Segre embedding. Identify $\mathbb{P}^{(k+1)(n+1)-1}$ with the projectivization $\mathbb{P}(\operatorname{Mat}(k+1, n+1))$ of the space of $(k+1) \times(n+1)$ matrices and consider the natural projection $\rho: \mathbb{P}(\operatorname{Mat}(k+1, n+1)) \rightarrow \mathbb{G}(k, n) \simeq \mathbb{G}(n-k-1, n)$. The following result is called the Cayley trick.

Theorem 1.14 [Gelfand et al. 1994; Weyman and Zelevinsky 1994]. The dual variety of $\mathbb{P}^{k} \times X$ coincides with the closure $\overline{\rho^{-1}(Z(X))}$, where

$$
Z(X) \subset \mathbb{G}(n-k-1, n)
$$

is the hypersurface defined by the Chow form of X.

The defining polynomial of the hypersurface $\overline{\rho^{-1}(Z(X))} \subset \mathbb{P}(\operatorname{Mat}(k+1, n+1))$ is called $X$-resultant; it provides another way of writing the Chow form of $X$.

Now, let $F_{0}, \ldots, F_{n}$ be $n+1$ generic homogeneous polynomials on $\mathbb{P}^{n}$ of the same degree $d>0$, and let $\mathbb{M}=\mathbb{M}\left(F_{0}, \ldots, F_{n}\right)$ be the $(n+1) \times N$ matrix of the coefficients of these polynomials, $N=\left(\begin{array}{c}n+d \\ n\end{array}\right)$. We consider the projection $\rho_{n, d}$ : $\mathbb{P}(\operatorname{Mat}(n+1, N)) \rightarrow \mathbb{G}(n, N-1) \simeq \mathbb{G}(N-n-2, N-1)$ defined by the maximal minors of $\mathbb{M}$.

Proposition 1.15 [Gelfand et al. 1994; Cox et al. 2005]. The hypersurface of degree $(n+1) d^{n}$ in $\mathbb{P}(\operatorname{Mat}(n+1, N))$ defined by the resultant $\operatorname{Res}\left(F_{0}, \ldots, F_{n}\right)$ coincides with the closure $\overline{\rho_{n, d}^{-1}\left(V\left(R_{n, d}\right)\right)}$, where $R_{n, d}$ denotes the Chow form of the $d$-th Veronese embedding of $\mathbb{P}^{n}$. In particular, $\operatorname{Res}\left(F_{0}, \ldots, F_{n}\right)$ is a polynomial in the maximal minors of $\mathbb{M}$.

2. IMPLEMENTATION. In this section, we illustrate briefly some of the methods available in the package Resultants, included with [Macaulay2]. We refer to the package documentation (which can be viewed with viewHelp Resultants) for more details and examples.

One of the main methods is resultant, which accepts as input a list of $n+1$ homogeneous polynomials in $n+1$ variables with coefficients in some commutative ring $A$ and returns an element of $A$, the resultant of the polynomials. There are 
no limitations on the ring $A$ because of Remark 1.3. The algorithms implemented are the Poisson formula (Theorem 1.7) and the Macaulay formula (Theorem 1.8). The former is used by default since it is typically faster, while for the latter one has to set the Algorithm option: resultant (...,Algorithm=>"Macaulay"). The method can also be configured to involve interpolation of multivariate polynomials (see [Manocha and Canny 1993]), i.e., it can reconstruct the polynomial resultant from its values at a sufficiently large number of points, which in turn are evaluated using the same formulas. The main derived method is discriminant, which applies the formula (1-5) to compute discriminants of homogeneous polynomials.

Example 2.1. In the following code, we take two forms $F, G$ of degree 6 on $\mathbb{P}^{3}$. We first verify that $\operatorname{Disc}(F)=0$ and $\operatorname{Disc}(G) \neq 0$ and then we compute the intersection of the pencil generated by $F$ and $G$ with the discriminant hypersurface in the space of forms of degree 6 on $\mathbb{P}^{3}$, which is a hypersurface of degree 500 in $\mathbb{P}^{83}$. (The algorithm behind these calculations is the Poisson formula; this is one of the cases where the Macaulay formula is much slower).

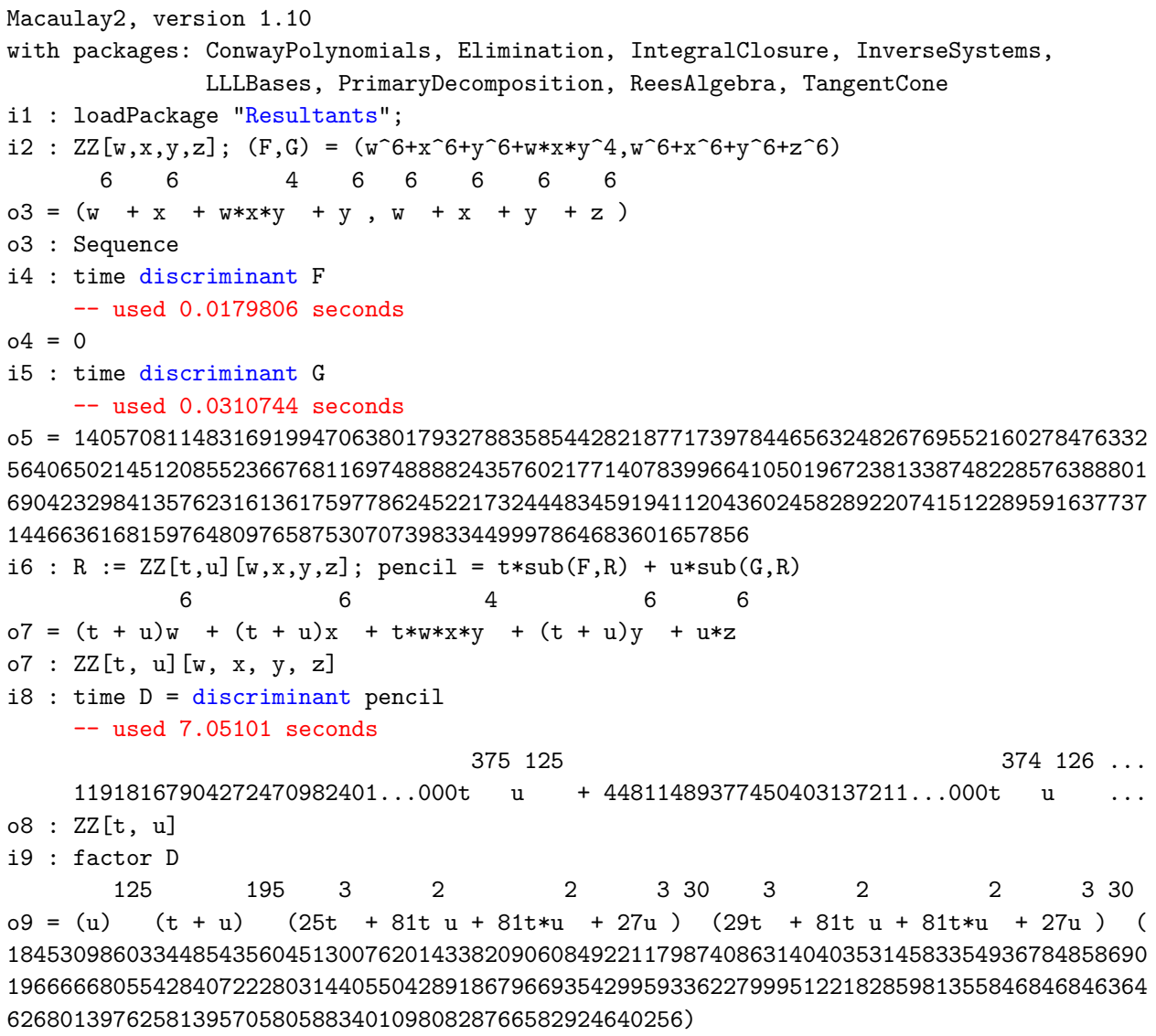
18453098603344854356045130076201433820906084922117987408631404035314583354936784858690 19666668055428407222803144055042891867966935429959336227999512218285981355846846846364 626801397625813957058058834010980828766582924640256)

o9 : Expression of class Product 
In particular, we deduce that the pencil $\langle F, G\rangle$ intersects the discriminant hypersurface in $F$ with multiplicity 125 , in $F-G$ with multiplicity 195 , and in another six distinct points with multiplicity 30 .

The package also provides methods for working with Chow forms and more generally tangential Chow forms of projective varieties (see [Gelfand et al. 1994, p. 104] and [Green and Morrison 1986]). In the following example, we apply some of these methods.

Example 2.2. Take $C \subset \mathbb{P}^{3}$ to be the twisted cubic curve.

i10 : C = kernel veronese $(1,3)$

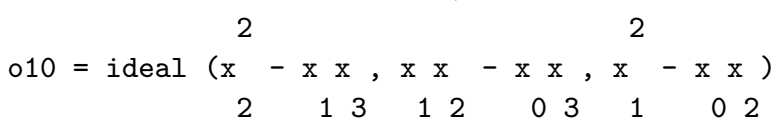

o10 : Ideal of $Q Q[x, x, x, x]$

$\begin{array}{llll}0 & 1 & 2 & 3\end{array}$

The Chow form of $C$ in $\mathbb{G}(1,3)$ can be obtained as follows:

i11 : $\mathrm{w}=$ chowForm $\mathrm{C}$

$$
\begin{aligned}
& 011=x^{3}-x+x+x+x^{2}+x^{2} x-2 x \quad x \quad x-x \quad x \quad x \\
& 1,2 \quad 0,21,21,3 \quad 0,1 \quad 1,3 \quad 0,22,3 \quad 0,1 \quad 1,22,3 \quad 0,1 \quad 0,32,3 \\
& \mathrm{QQ}[\mathrm{x}, \mathrm{x}, \mathrm{x}, \mathrm{x}, \mathrm{x}, \mathrm{x}] \\
& 0,1 \quad 0,2 \quad 1,2 \quad 0,3 \quad 1,3 \quad 2,3
\end{aligned}
$$

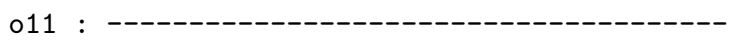

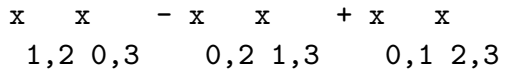

We can recover $C$ from its Chow form by taking the so-called Chow equations; see [Gelfand et al. 1994, p. 102; Catanese 1992].

i12 : $\mathrm{C}==$ saturate chowEquations $\mathrm{w}$

012 = true

The $X$-resultant of $C$ can be obtained applying first the duality isomorphism $\mathbb{G}\left(1, \mathbb{P}^{3}\right)=\mathbb{G}\left(1, \mathbb{P}^{3^{*}}\right)$ and then passing from the Plücker to the Stiefel coordinates.

$$
\begin{aligned}
& \text { i13 : } \mathrm{w}^{\prime}=\text { dualize } \mathrm{w}
\end{aligned}
$$

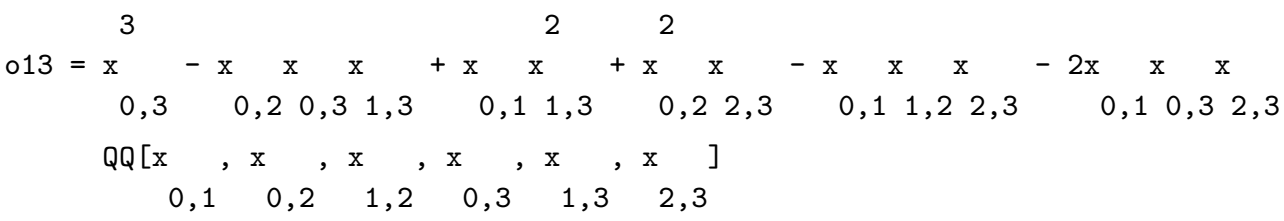

$$
\begin{aligned}
& 1,20,3 \quad 0,21,3 \quad 0,12,3
\end{aligned}
$$

i14 : fromPluckerToStiefel w' 


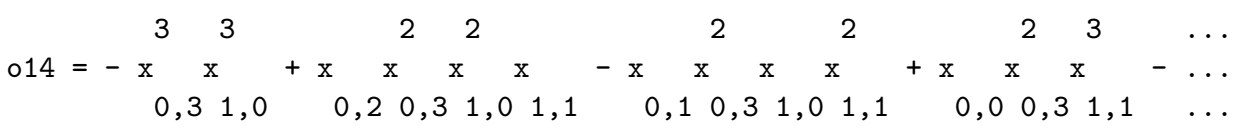

$014: \mathrm{Q}[\mathrm{x}, \mathrm{x}, \mathrm{x}, \mathrm{x}, \mathrm{x}, \mathrm{x}, \mathrm{x}, \mathrm{x}]$

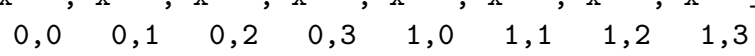

The method cayleyTrick returns a pair consisting of the defining ideal of $\mathbb{P}^{1} \times C \subset \mathbb{P}^{7} \simeq \mathbb{P}(\operatorname{Mat}(2,4))$ and the $X$-resultant of $C$, considered as a hypersurface $Z \subset \mathbb{P}(\operatorname{Mat}(2,4))$. Theorem 1.14 ensures that $Z$ is the dual variety of $\mathbb{P}^{1} \times C$. We can check this using the method dualVariety.

i15: $(\mathrm{P} 1 \mathrm{xC}, \mathrm{Z})$ = cayleyTrick $\mathrm{C}$;

i16 : dualVariety $(\mathrm{P} 1 \mathrm{xC})==\mathrm{Z}$

$016=$ true

Some overlapping packages. There are two further packages related to resultant computations, which are included in Macaulay2: [Elimination] by M. E. Stillman, and [EliminationMatrices] by N. Botbol, L. Busé and M. Dubinsky. The former contains functions to compute Sylvester resultants. The latter can compute different resultant matrices; in particular, it contains an implementation of the Macaulay formula.

A further package for working with Chow forms is Coisotropy, by K. Kohn (see [Kohn 2016]), which, in particular, contains a useful function to compute the degrees of all tangential Chow forms of a given projective variety.

For all these overlapping functions, it does not seem easy to rank implementations in terms of efficiency because this generally depends on the problem. They also differ in how they handle input and output. For instance, the discriminant of a binary form computed using the package Elimination lies again in the same ring, rather than in the ring of coefficients, and the Chow form of a projective variety computed using Coisotropy lies in a polynomial ring, rather than in a quotient ring.

SUPPLEMENT. The online supplement contains version 1.2.1 of Resultants.

\section{REFERENCES.}

[Bajaj et al. 1988] C. Bajaj, T. Garrity, and J. Warren, "On the applications of mulit-equational resultants", tech report 88-826, Purdue University, 1988.

[Busé and Jouanolou 2014] L. Busé and J.-P. Jouanolou, "On the discriminant scheme of homogeneous polynomials", Math. Comput. Sci. 8:2 (2014), 175-234. MR Zbl

[Catanese 1992] F. Catanese, "Chow varieties, Hilbert schemes and moduli spaces of surfaces of general type”, J. Algebraic Geom. 1:4 (1992), 561-595. MR Zbl

[Cox et al. 2005] D. A. Cox, J. Little, and D. O'Shea, Using algebraic geometry, 2nd ed., Graduate Texts in Mathematics 185, Springer, 2005. MR Zbl

[Cox et al. 2007] D. Cox, J. Little, and D. O'Shea, Ideals, varieties, and algorithms: An introduction to computational algebraic geometry and commutative algebra, 3rd ed., Springer, New York, 2007.

MR Zbl 
[Demazure 2012] M. Demazure, "Résultant, discriminant", Enseign. Math. (2) 58:3-4 (2012), 333373. MR Zbl

[Elimination] M. E. Stillman, "Elimination", Macaulay2 package, 2005, available at http://github.com/ Macaulay2/M2/blob/master/M2/Macaulay2/packages/Elimination.m2.

[EliminationMatrices] N. Botbol, L. Busé, and M. Dubinksy, "EliminationMatrices", Macaulay2 package, 2012, available at http://github.com/Macaulay2/M2/blob/master/M2/Macaulay2/packages/ EliminationMatrices.m2.

[Elkadi and Mourrain 1998] M. Elkadi and B. Mourrain, "Some applications of Bezoutians in effective algebraic geometry”, INRIA, 1998, available at https://hal.inria.fr/inria-00073109.

[Emiris and Mourrain 1999] I. Z. Emiris and B. Mourrain, "Matrices in elimination theory", J. Symbolic Comput. 28:1-2 (1999), 3-44. MR Zbl

[Gelfand et al. 1994] I. M. Gelfand, M. M. Kapranov, and A. V. Zelevinsky, Discriminants, resultants, and multidimensional determinants, Birkhäuser, Boston, 1994. MR Zbl

[Green and Morrison 1986] M. L. Green and I. Morrison, "The equations defining Chow varieties", Duke Math. J. 53:3 (1986), 733-747. MR Zbl

[Jouanolou 1991] J.-P. Jouanolou, "Le formalisme du résultant", Adv. Math. 90:2 (1991), 117-263. MR Zbl

[Jouanolou 1997] J. P. Jouanolou, "Formes d'inertie et résultant: un formulaire", Adv. Math. 126:2 (1997), 119-250. MR Zbl

[Kapur et al. 1994] D. Kapur, T. Saxena, and L. Yang, "Algebraic and geometric reasoning using Dixon resultants", pp. 99-107 in Proceedings of the International Symposium on Symbolic and Algebraic Computation, ACM, New York, NY, USA, 1994. Zbl

[Kohn 2016] K. Kohn, "Coisotropic hypersurfaces in grassmannians”, 2016. arXiv

[MacAulay 1903] F. S. MacAulay, "On some formulae in elimination”, Proc. Lond. Math. Soc. 35 (1903), 3-27. MR Zbl

[Macaulay2] D. R. Grayson and M. E. Stillman, "Macaulay2, a software system for research in algebraic geometry", software, version 1.10, available at http://faculty.math.illinois.edu/Macaulay2.

[Manocha and Canny 1993] D. Manocha and J. F. Canny, "Multipolynomial resultant algorithms", J. Symbolic Comput. 15:2 (1993), 99-122. MR Zbl

[van der Waerden 1950] B. L. van der Waerden, Modern algebra, vol. II, Ungar, New York, 1950. Reprinted Springer, 1991, as Algebra, vol. II. MR

[Weyman and Zelevinsky 1994] J. Weyman and A. Zelevinsky, "Multiplicative properties of projectively dual varieties”, Manuscripta Math. 82:2 (1994), 139-148. MR Zbl

\section{ReCEIVED: 3 May 2017 ReVised: 26 Jan 2018 ACCEPTED: 18 May 2018}

\section{GIOVANNI STAGLIANÒ:}

giovannistagliano@gmail.com

Dipartimento di Ingegneria Industriale e Scienze Matematiche, Università Politecnica delle Marche, Ancona, Italy 

HeLP: a GAP package for torsion units in integral group rings

Andreas Bächle and Leo Margolis

A software package to compute automorphisms of graded algebras

Simon Keicher

A package for computations with classical resultants

Giovanni Staglianò

The Space Curves package in Macaulay2

Mengyuan Zhang

The ReesAlgebra package in Macaulay2

David Eisenbud

A Macaulay2 package for computations with rational maps

Giovanni Staglianò

ExteriorIdeals: a package for computing monomial ideals in an exterior algebra

Luca Amata and Marilena Crupi

Software for computing conformal block divisors on $\bar{M}_{0, n}$

David Swinarski

Divisor Package for Macaulay2

Karl Schwede and Zhaoning Yang 